Annuaire suisse de politique de développement

2 | 1982

Annuaire Suisse - Tiers Monde 1982

\title{
2. Politique économique extérieure
}

\section{Hilmar Stetter}

\section{OpenEdition}

\section{Journals}

Édition électronique

URL : http://journals.openedition.org/aspd/1119

DOI : $10.4000 /$ aspd. 1119

ISSN : 1663-9669

\section{Éditeur}

Institut de hautes études internationales et du développement

\section{Édition imprimée}

Date de publication : 31 août 1982

Pagination : 61-80

ISSN : 1660-5934

\section{Référence électronique}

Hilmar Stetter, «2. Politique économique extérieure », Annuaire suisse de politique de développement [En ligne], 2 | 1982, mis en ligne le 20 janvier 2013, consulté le 08 septembre 2020. URL : http:// journals.openedition.org/aspd/1119; DOI : https://doi.org/10.4000/aspd.1119 


\section{POLITIQUE ECONOMIQUE EXTERIEURE}

\section{LA POLITIQUE DE DEVELOPPEMENT AU PARLEMENT}

La réduction de 18\% du budget 1982 de la coopération au développement, l'acceptation de diverses mesures influençant les échanges commerciaux et la politique des matières premières, la continuation de l'aide humanitaire internationale, telles ont été l'an passé les décisions importantes prises par le Parlement en matière de développement. Le Conseil national a également accepté que se poursuive le financement de diverses mesures économiques et commerciales. Au Conseil des Etats comme au Conseil national, diverses interventions personnelles ont mis en lumière certains aspects de la politique suisse de développement.

Nous passons ici en revue les dossiers majeurs de la politique de développement dont le Parlement s'est occupé de juillet 1981 à juin 1982.

\section{Messages et rapports}

Aux termes du message sur les mesures touchant aux échanges commerciaux et au commerce des matières premières dans l'optique du développement, texte daté du 25 février 1981, le Parlement a décidé à l'unanimité le 9 octobre 1981 de continuer d'accorder les préférences douanières aux pays en développement; il a accepté les accords sur la création d'un Fonds commun pour les produits de base et celui sur le caoutchouc naturel, accords auxquels la Suisse va adhérer. II en va de même pour l'accord international sur le cacao de 1980 (1).

En vertu du message du 27 mai 1981 sur la contribution ordinaire de la Confédération au Comité international de la Croix-Rouge, le Parlement a décidé le 1er décembre de porter cette contribution à 20 millions de francs par an (2). Aux termes du message du 27 mai 1981 sur un crédit-cadre permettant de poursuivre le financement de l'aide humanitaire internationale, le Parlement a décidé à l'unanimité le 3 décembre 1981 d'octroyer 360 millions de francs pour financer durant trois ans au minimum des mesures 
d'aide humanitaire et alimentaire (3).

Les coupes pratiquées par le Parlement dans le budget de la Confédération pour 1982 ont entraîné des réductions budgétaires pour la coopération au développement. Les dépenses de tous les départements ont été réduites de 10\% en vertu des économies décidées par la Confédération le 20 juin 1980. Afin de réduire le déficit budgétaire, les montants mis à la disposition de la coopération au développement ont subi un abattement supplémentaire de $8 \%$. Les prestations de la coopération au développement piétinent ainsi aux environs de $0,25 \%$ du PNB et l'augmentation en chiffres réels qui avait été prévue n'a pas été atteinte.

Lors de la discussion du budget, le Conseil des Etats refusa de supprimer l'abattement supplémentaire de $8 \%$ infligé à la coopération au développement (proposition Bauer-Lagier, PL, GE) et le Conseil national refusa de réduire de moitié la portée de cette mesure (proposition Ott, PS, BL) (4).

Le 23 juin 1982, le Conseil national accepta à l'unanimité la décision du Conseil fédéral concernant le financement des mesures économiques et commerciales dans l'optique de la coopération au développement. II s'agit d'un crédit-cadre de 350 millions de francs pour une durée minimum de trois ans. Le message du Conseil fédéral y relatif est daté du 14 décembre 1981 (5).

Le 25 juin, le Parlement décida à l'unanimité d'accepter le projet de loi sur les mesures touchant au commerce extérieur. Le même jour, il décida toujours à l'unanimité de promulguer une loi fédérale sur la contribution annuelle à verser à l'Office suisse d'expansion commerciale (OSEC), décision basée sur le message du 11 novembre 1981. Autre décision unanime du Conseil national concernant I'adhésion de la Suisse au sixième accord international sur I'étain en 1981.

\section{Initiatives populaires}

Le 17 juin, le Conseil national a décidé par 71 voix contre 68 de recommander au peuple et aux cantons le rejet de l'initiative populaire "contre les importations exagérées de fourrages et les 'usines à viande' et pour une meilleure utilisation du sol national" (initiative sur les fourrages). La loi sur l'agriculture n'en devrait pas moins être révisẹe (6).

\section{Motions}

Le 7 octobre 1981, le Conseil des Etats a traité de la motion Schmid (PDC, Al) sur "I'aide au développement". Le conseiller aux Etats Schmid avait présenté cette motion en décembre 1980. II souhaitait une modification de la loi sur la coopération au développement permettant une action parlementaire accrue dans ce domaine. II désirait que :

1. les mesures prises par la Confédération dans le domaine de la coopération technique, de l'aide financière et de l'aide humanitaire régulière fussent inscrites sur des programmes annuels et soumises à l'approbation des 
Chambres fédérales;

2. les montants nécessaires au financement de ces programmes fussent accordés sous la forme d'un crédit global.

Après étude de la motion, la commission des affaires étrangères du Conseil des Etats en vint à la conclusion que la motion ne pouvait être présentée sous sa forme première et que, si elle n'était pas retirée, il y aurait lieu d'en recommander le rejet par l'assemblée. Pour donner suite aux vœux exprimés dans la motion, le Conseil fédéral fit savoir qu'il prévoyait les mesures suivantes :

1. "Nous adresserons aux commissions des affaires étrangères un rapport relativement détaillé sur tout projet qui impliquera une dépense supérieure à 500.000 francs.

2. Nous avons l'intention de vous soumettre au moins une fois par année un 'tour d'horizon'. Ce nouveau rapport sera intituté 'Tour d'horizon en matière d'aide publique au développement'. Ce document vous informera sur tous les domaines de notre coopération.

3. Nous nous proposons en outre de faire un effort de clarification dans la présentation du budget. II nous permettra de vous donner, dans le cadre du budget, des informations beaucoup plus détaillées que celles que nous pouvions vous donner jusqu'ici.'

En vertu de ces déclarations, la motion Schmid fut retirée (7).

\section{Postulats}

Le conseiller aux Etats Generali (PR, TI) a présenté le 19 mars 1981 un postulat qui demande un rapport sur les conséquences de l'aide au développement pour l'économie suisse. En acceptant ce postulat le 7 octobre 1981, le conseiller fédéral Honegger fit remarquer qu'en 1980 l'aide publique de la Suisse s'était élevée à quelque 405 millions de francs. Les marchandises et les services nécessaires à la réalisation des projets sont en général fournis sous une forme non liée, c'est-à-dire que leur lieu d'origine n'est pas forcément la Suisse. Les marchandises et les services fournis en 1980 par l'économie suisse au titre de la coopération publique au développement ont atteint une valeur de 337 millions de francs qui passe à 551 de francs si I'on tient compte des livraisons faites à des projets de la Banque mondiale. Cette statistique est tenue à jour (8).

Les postulats suivants ont été présentés au Conseil national de juillet 1981 à juin 1982 :

- Postulat Morf (PS, ZH), aide aux femmes des pays en développement : le Conseil fédéral fera rapport sur ses efforts pour contribuer à l'amélioration de la condition féminine dans les pays en développement et dans le cadre de la coopération suisse au développement (9 octobre 1981) (9).

- Postulat Martin (PR, VD), coopération technique : le Conseil fédéral étudiera la possibilité d'offrir, dans le cadre de la coopération technique, des chances de formation pratique à des ingénieurs ayant achevé leurs 
études (9 octobre 1981) (10).

- Postulat Petitpierre (PR, GE), aide en cas de catastrophes et service militaire : dans le cadre de l'aide en cas de catastrophes, le Conseil fédéral est prié d'assimiler à des prestations de service militaire les stages des volontaires; le postulat Wellauer (PDC, TG), renforcement du corps d'aide en cas de catastrophe, lui demande d'étudier la possibilité de faire intervenir à l'étranger des détachements de l'armée au titre de l'aide en cas de catastrophes (9 octobre 1981) (11).

\section{Interpellations}

Les relations entre la Suisse et le Tiers Monde ont également fait l'objet de diverses interpellations au Conseil national en 1981-82. Dans une interpellation de la fraction radicale, à l'enseigne de la "politique de développement", on s'en prit aux critiques dirigées par la gauche contre la politique de développement du gouvernement, on voulut savoir quelles étaient les principales difficultés actuelles et on exprima le vœu de débats plus soutenus concernant le développement. Dans sa réponse, le Conseil fédéral indiqua que "les forces politiques dominantes du pays soutiennent toujours l'action du gouvernement en matière de développement". Les principales difficultés de la politique gouvernementales proviennent de la modestie des ressoures financières disponibles. Au demeurant, le Conseil fédéral pense "qu'une information libérale et si possible complète est la meilleure garantie de l'approbation par la population de la politique suivie" (9 octobre 1981) (12). Cette interpellation a fait l'objet d'une longue discussion au cours de la session d'été, le 24 juin 1982.

La fraction PdT/PSA/POCH a demandé par une interpellation "Crédit pour le Maroc" le renvoi de la ratification d'un crédit mixte de plus de 55 millions de francs conclu en 1981, et cela en raison de la permanence du conflit entre le Maroc et les Sahraoui. II y a là une atteinte aux droits de l'homme qui contrevient à nos critères d'aide au développement. Dans sa réponse, le Conseil fédéral fait remarquer que le crédit en question ne peut être repoussé que si les tensions s'aggravent au point de compromettre les objectifs du développement visés par notre contribution, ce qui n'est pas le cas au Maroc. Le Conseil fédéral n'estime donc pas nécessaire de renvoyer à plus tard la ratification du crédit mixte déjà cité (9 octobre 1981) (13).

L'interpellation Biel (Ind., ZH) "Suisses d'Algérie, indemnisation" s'enquiert de l'état des négociations concernant un accord d'indemnisation avec l'Algérie, où les biens de citoyens suisses de l'étranger ont été nationalisés; la Confédération est-elle véritablement en mesure de verser des avances aux personnes lésées? Le Conseil fédéral a répondu que l'Algérie n'était pas disposée à poursuivre les négociations concernant une indemnisation et que la caisse fédérale ne pourrait pas indemniser les pertes subies. Les mesures d'assistance qui s'imposaient ont cependant été prises (9 octobre 1981) (14).

L'interpellation Leuenberger (PS, ZH) "Artistes du Tiers Monde" demandait au Conseil fédéral s'il était disposé à retirer aux termes de la loi la qualité 
d'artistes aux "go-go-girls" et à modifier la pratique actuelle qui permet d'accorder à ces jeunes femmes des autorisations de séjour hors contingentement. Dans sa réponse, le Conseil fédéral fait remarquer que $34 \%$ des autorisations accordées concernent des non-Européennes; il pense que "le fait que les 'go-go-girls' soient assimilées à des artistes échappant à la limitation des autorisations a engendré une situation déplaisante". A l'avenir, les "go-go-girls" doivent être soumises au contingentement. Lors de l'octroi de l'autorisation, il y a lieu de contrôler les clauses de l'engagement et les conditions de logement. Avant de passer la frontière, les "go-yo-girls" doivent être informées avec précision du travail qui les attend (18 décembre 1981) (15).

La fraction socialiste a demandé dans son interpellation "Politique étrangère" que soient renforcées la coopération au développement et l'aide économique en faveur des pays en développement qui doivent affronter un endettement toujours plus grave.

Dans une réponse écrite, le Conseil fédéral a fait remarquer qu'il y avait toute une série de pays en développement jouissant d'un certain essor économique et connaissant un déséquilibre de la balance des paiements et l'endettement. S'ils entendent réussir l'indispensable réforme de leurs structures économiques, ces pays doivent en appeler un certain temps à un financement venant de l'étranger. Dans le meilleur des cas, cet apport ne peut être fourni que partiellement par l'aide publique au développement. C'est donc au système bancaire international à entrer le premier en action, mais ses prestations sont complétées à un rythme croissant par des initiatives relevant de l'aide économique internationale. Dans la deuxième moitié de la présente législature, le Conseil fédéral établira, comme prévu, une base juridique pour la participation de la Suisse aux initiatives de l'aide économique internationale (8 mars 1982) (16).

Une interpellation de la fraction socialiste "Aide économique à la Turquie" demandait la suspension du crédit d'aide économique de 35 millions de francs tant qu'on ne verrait aucun progrès du processus de démocratisation et proposait le refus de tous nouveaux crédits. Le Conseil fédéral répondit que le crédit en question faisait partie d'une opération d'assistance 1980 de I'OCDE toujours en cours. Le Parlement a ratifié I'accord bilatéral signé à ce sujet. En droit international, cet accord est contraignant et ne peut être ni suspendu ni révoqué de manière unilatérale. La situation politique intérieure en Turquie est source de préoccupation. A moyen terme, on peut espérer un assainissement économique (24 juin 1982) (17). Une interpellation Herczog $(\mathrm{POCH}, \mathrm{ZH})$ "Aide économique à Turquie, suspension" fit l'objet d'une réponse identique (19 mars 1982) (18).

Le conseiller national Linder (LIB, BS) avait déposé une interpellation "Aide au développement, Institut suisse des tropiques" pour s'informer des conditions de financement de I'Institut suisse des tropiques par la DDA. Le Conseil fédéral fit savoir dans sa réponse que la DDA avait augmenté le mon- 
tant de sa contribution, mais que les déficits croissants de l'Institut devraient être examinés par toutes les instances intéressées (canton et administration fédérale) (24 juin 1982) (19). Les relations entre la Suisse et le Tiers Monde suscitèrent encore diverses simples questions ou furent évoquées au cours des heures réservées aux libres questions.

\section{CONTRIBUTION FEDERALE A L'OFFICE SUISSE D'EXPANSION COMMERCIALE (OSEC)}

C'est au moyen d'une modification de la loi que le Conseil fédéral entend subventionner à l'avenir l'Office suisse d'expansion commerciale (OSEC) qui recevra 7 millions de francs par an ou au maximum $45 \%$ du total de ses dépenses. Les prestations ont été irrégulières jusqu'ici, représentant ces dernières années environ $45 \%$ du total des dépenses de l'institution. L'OSEC s'occupe aussi de l'expansion des importations, secteur récemment renforcé. Le 25 juin 1982, la nouvelle loi a été acceptée sans opposition par le Parlement. Elle entrera en vigueur le 1er janvier 1983 (20).

L'OSEC est une association privée qui compte 2.000 adhérents recrutés dans les milieux de l'exportation; il s'occupe de promouvoir les exportations suisses et de faire connaître l'économie nationale. II organise des foires et des expositions industrielles, fournit des conseils et de la documentation sur les marchés étrangers.

Parmi les foires et expositions des pays en développement qui ont retenu I'attention de I'OSEC, il importe de citer Tecno Suiza, un symposium technologique réalisé en collaboration avec l'ambassade de Suisse et la Chambre suisse du commerce en Argentine. Tecno Suiza a eu lieu à Buenos-Aires, du 9 au 13 novembre 1981.

Le Service d'information tiers monde a suggéré qu'une promotion officielle des exportations vers les pays en développement devrait au moins tenir compte des principes généraux de la loi fédérale sur la coopération au développement. Car la promotion des exportations ne va pas fatalement dans le même sens que l'effort de développement de ces pays (21).

On n'a jamais disposé de grands moyens pour promouvoir l'expansion commerciale des pays en développement; rares ont été les informations concernant le marché suisse. Un contrat spécial avec I'OFAEE règle les conditions de la promotion des importations, la compétence de I'OSEC en la matière étant soulignée dans le message du Conseil fédéral. L'OSEC doit s'en occuper dans le cadre de la coopération au développement et il reçoit un crédit spécial à cet effet. Les opérations sont financées par le crédit-cadre pour les mesures économiques et commerciales à adopter en faveur des pays en développement. Pour inaugurer ce premier contrat de deux ans, I'OSEC a commencé de faire 
paraître un bulletin des importations. Cette publication contient des offres de produits des pays en développement à destination du marché suisse.

\section{LOI FEDERALE SUR LES MESURES TOUCHANT AUX RELATIONS ECONOMIQUES AVEC L'ETRANGER}

En juillet 1981, le Conseil fédéral proposait une loi sur les mesures touchant aux relations économiques avec l'étranger, afin de prendre le relais de l'arrêté fédéral, limité dans le temps, sur diverses mesures d'ordre économique (comme la surveillance des importations de textiles, les importations de fourrages). Le message correspondant a pris valeur définitive au 25 juin 1982 et a été accepté sans opposition par le Parlement.

La loi permet au Conseil fédéral de prendre des mesures défensives quand des intérêts économiques suisses essentiels sont compromis "par des décisions étrangères ou des circonstances extraordinaires à l'étranger" dans les domaines du trafic des marchandises et des paiements ou de la prestation de services. Cette extension de la loi aux prestations de services est nouvelle; l'économie la désirait et le Conseil fédéral ne l'avait pas prévue à l'origine. Avec certains pays, il n'existe aucune réglementation pour le trafic des paiements, mais des restrictions ne sont pas impensables compte tenu de l'endettement considérable de bien des pays (22).

Au cours de la procédure de consultation, I'Union syndicale suisse proposa de prendre aussi des mesures défensives contre le "dumping social", mesures à adopter au moment où "de fortes limitations de la liberté de coalition" à l'étranger viendraient à menacer "I'emploi ou le niveau des salaires" en Suisse.

Cet élargissement du texte visait la possibilité de limiter les importations de marchandises bon marché produites et exportées par des Etats dictatoriaux où les salaires sont maintenus artificiellement bas et où les libertés syndicales n'existent pas (23).

Pour sa part, la communauté de travail Swissaid/Pain pour le prochain/Action de Carême/Helvetas souhaitait aussi des mesures défensives dans les cas où les objectifs essentiels de la sécurité et de la politique suisse de développement seraient compromis. Lors de conflits, il s'agirait de ne pas se préoccuper uniquement des intérêts de l'économie suisse, mais aussi de ce qui touche au développement et à la sécurité (24).

Lors de l'examen de la loi par le Conseil national (18 mars 1982), la proposition Jaggi (PS, VD) qui reprenait les postulats syndicaux, fut repoussée tout comme les propositions Blunschy (PDC, SZ) qui s'occupaient des incidences de la loi sur la politique de développement (25). Invoquant la poursuite d'une politique libérale des échanges internationaux, le Conseil fédéral 
ne se montra pas disposé à inclure dans la nouvelle loi ces propositions complémentaires, selon lui susceptibles d'engendrer un protectionnisme accru. II vaudrait mieux selon lui, du point de vue économique, améliorer la division internationale du travail.

\section{CREDIT-CADRE POUR L'AIDE ECONOMIQUE INTERNATIONALE}

L'Office fédéral des affaires économiques extérieures (OFAEE) ayant orienté la commission de la politique commerciale et la commission de la coopération internationale au développement sur le projet de création d'un créditcadre de $\mathbf{5 0 0}$ millions de francs pour l'aide économique internationale, les plans établis furent communiqués à I'opinion publique en septembre 1981.

Ce crédit-cadre servira à financer des interventions en faveur des pays affectés par un déséquilibre de leur balance des paiements. 400 millions de francs constitueront la garantie des crédits ouverts par des banques privées et 100 millions iront directement dans les pays intéressés au titre des subventions pour le paiement des intérêts. La Confédération, les banques et l'économie d'exportation épauleraient ces opérations de crédit. "Les banques doivent accorder des conditions favorables aux pays nécessitant un assainissement financier des crédits garantis par la Confédération; le paiement des intérêts étant normalement un risque qui leur incombe. Les montants fournis par la Confédération ne doivent cependant être utilisés que pour ouvrir de nouveaux crédits. Dans la mesure du possible, ces crédits devraient être liés à des livraisons de marchandises suisses. L'économie d'exportation doit aussi assumer une partie des risques." Un "fonds de garantie et de couverture des pertes" identique à la GRE serait enfin créé. Les bénéficiaires de cette aide économique sont des Etats industriels financièrement faibles ou des pays parvenus au seuil du développement. Dans l'intervalle, le Conseil fédéral a différé la publication de son message sur le crédit-cadre projeté (26).

L'Union syndicale suisse a émis des critiques peu après la communication du projet, arguant surtout du fait que les compétences politiques se trouvaient modifiées. C'était jusqu'ici le seul Parlement qui avait à se prononcer sur les mesures d'aide économique. L'USS faisait allusion à l'état précaire des finances fédérales et critiquait le fait que la Confédération dut intervenir après que les banques eurent contribué à l'endettement des pays intéressés en leur accordant des crédits (27).

Dans les milieux qui militent pour le développement, la Déclaration de Berne a déjà fait savoir qu'un tel crédit-cadre pourrait être combattu au moyen du référendum. La communauté de travail Swissaid/Pain pour le prochain/Action de Carême/Helvetas a annoncé qu'elle allait de toute façon étudier le recours au référendum, d'autant plus que le crédit-cadre envisagé 
lui paraît devoir déplacer des pays en développement les plus pauvres vers les mieux lotis les faibles ressources financières dont dispose la coopération au développement (28).

\section{AIDE ECONOMIQUE A LA TURQUIE}

En 1980, la Suisse a accordé à la Turquie un crédit d'aide économique de 35 millions de francs. L'accord à ce sujet est entré en vigueur le 23 septembre 1981. II porte sur les fournitures de marchandises et de services d'origine suisse jusqu'au 30 juin 1982, date portée au contrat. L'économie turque n'ayant que partiellement utilisé ce crédit dans le délai prescrit, l'accord a été prolongé d'un an, jusqu'au 30 juin 1983 (29).

Au cours de l'automne 1981, deux interpellations ont été présentées au Conseil national. On y demandait au Conseil fédéral de surseoir à l'aide accordée à la Turquie par le Parlement et cela jusqu'au moment où le régime militaire turc aurait pris les premières mesures d'un retour à la démocratie. Le Conseil fédéral expliqua que l'accord intervenu était contraignant en droit international et en voie d'application. II ne saurait être question de bloquer I'aide économique. Lors d'autres mesures d'aide économique prises dans le cadre de l'OCDE en 1981, la Suisse s'est montrée réservée et n'a pas pris d'engagements concrets. Il lui aurait fallu d'autres bases légales pour pouvoir le faire (30).

\section{GARANTIE CONTRE LES RISOUES A L'EXPORTATION}

En 1981, la garantie contre les riques à l'exportation (GRE) a enregistré un nouveau déficit arrêté à 123,03 millions de francs. Ses réserves sont tombées à 77,4 millions de francs. La GRE n'en doit pas moins travailler à longue échéance avec ses propres fonds. Elle assure les envois des exportateurs suisses contre les risques politiques, les défauts de fabrication ou les fluctuations monétaires. La garantie couvre au maximum $95 \%$ de la valeur de la livraison, contre paiement d'une taxe adéquate. Dans la règle, les risques sont couverts dans une proportion de 70 à $90 \%$.

La GRE a connu en 1981 sa première année d'autonomie financière, une année où l'assurance contre les risques monétaires a de nouveau renchéri. Le Conseil fédéral a pris diverses mesures qui contraignent les assurés à assumer une part plus forte des risques monétaires.

Près de 4.800 demandes ont été acceptées en 1981, la somme des engagements 
s'élevant à fin 1981 à 22,3 milliards de francs (25,3 milliards de francs en 1980). L'industrie des machines est classée en tête de liste.

$\begin{array}{lc}\text { La GRE en chiffres (à fin 1981) } & \text { milliards de francs } \\ \begin{array}{l}\text { Total des montants garantis } \\ \text { Montant des garanties vis-à-vis }\end{array} & 22,3 \\ \begin{array}{l}\text { de tous les pays en développement } \\ \text { Montant des garanties vis-à-vis }\end{array} & 14,65 \\ \begin{array}{l}\text { des pays les moins avancés } \\ \text { Réserves }\end{array} & 0,187 \\ \end{array}$

Le montant des garanties pour des livraisons à des pays en développement s'élève à 14.654 millions de francs, ce qui représente une proportion de $65,7 \%$ du total des garanties. $36,8 \%$ concernent l'Asie, 22,5\% I'Amérique latine, 25,8\% l'Afrique et $14,9 \%$ des pays en développement d'Europe.

Si les pays en développement représentent globalement la plus grande partie des livraisons sous garantie, la part des cinquante-deux pays classés par I'OCDE dans la catégorie des faibles revenus est modeste. Ils n'ont requis que $8,4 \%$ du total des garanties, soit 1.870 millions de francs. Pour sept d'entre eux, les engagements contractés ont dépassé 50 millions de francs par pays; pour quatorze autres, les garanties étaient de plus de 20 millions de francs. Les livraisons sont garantie ont été fortement orientées vers les six pays bénéficiant d'un crédit mixte de la Suisse ou d'un soutien à leur balance des paiements (Egypte, Sénégal, Cameroun, Kenya, Soudan et Tanzanie). Ces six pays ont enregistré $51 \%$ des garanties couvrant les envois aux pays en développement à faible revenu. II faut tenir compte ici de la modification de la loi sur la GRE et des principes de la politique de développement adoptée par la Suisse. Les remboursements des pertes subies se sont élevés à 301,5 millions de francs, dont 192,8 millions de francs pour pertes de change. Vient en second rang, avec 91 millions de francs, la consolidation des dettes de la Turquie, du Togo, du Pérou, du Zaïre et du Soudan (31).

Par une simple question du 14 décembre 1981, Mme Blunschy, conseillère nationale (PDC, SZ) s'est enquis des expériences faites lors des exportations vers les pays les moins avancés, compte tenu des critères de la politique suisse de développement. Les informations récentes en provenance de ces pays et le volume des garanties qui les concernent sont évidemment à l'origine de cette intervention. Dans sa réponse, le Conseil fédéral indique que les critères déterminants dans I'optique du développement sont "la situation financière actuelle, le degré de l'endettement et le potentiel de développement du pays de destination". Pour le reste, ce sont les principes généraux de la politique de développement qui entrent en jeu (32).

Le service d'information $\mathrm{i} 3 \mathrm{~m}$ voudrait soumettre la GRE à diverses autres 
règles spécifiques $(33)$ :

- respect des principes généraux de notre politique de développement même dans le cas où ils entrent en conflit avec la création d'emplois en Suisse et les conditions générales d'assurance;

- extension aux livraisons faites à tous les pays en développement du respect des principes généraux de notre politique de développement;

- élargissement de la commission de la GRE;

- amélioration des processus de décision et de l'information;

- plus de transparence et meilleur contrôle des opérations.

Ajoutons que, dans sa réponse à une simple question du conseiller national Crevoisier (PSA, BE), le Conseil fédéral a annoncé "qu'en raison des riques encourus au Togo, aucune GRE ne serait accordée à un projet de développement agricole doté d'industries de transformation, projet assumé à la demande du gouvernement togolais par une firme suisse d'ingénieurs" (34).

Le Conseil fédéral s'attend en 1982 à un excédent de charges de la GRE d'environ 30 millions de francs, que le reliquat des réserves ne couvrira pas. La Confédération devra fournir une avance portant intérêt. Des excédents de dépenses sont également prévus ces prochaines années (35).

\section{GARANTIE AUX RISQUES DE L'INVESTISSEMENT}

En 1981, la garantie aux risques de l'investissement a été sollicitée davantage que les années précédentes. Six investissements ont bénéficié d'une garantie totale de 38,9 millions de francs. Trois investissements concernent le Togo (machines, agriculture), les autres la Yougoslavie (machines), la Malaisie (textiles) et les Philippines (montres). La garantie de huit autres investissements est envisagée. A fin 1981, les engagements contractés par la Confédération s'élevaient à 34,6 millions de francs, dont $41 \%$ concernaient des pays en développement africains, $40 \%$ des pays européens et $19 \%$ des pays asiatiques. Les remboursements se sont élevés à 24 millions de francs. Deux compensations de pertes subies ont pu être couvertes par la perception des taxes courantes (36).

II y a lieu de rappeler que le montant de chaque garantie et le nom de I'entreprise suisse intéressée sont de nature confidentielle. Les principes généraux de la politique de développement de la Confédération doivent être observés lors de I'octroi de la garantie par la commission de la garantie aux risques de I'investissement. 


\section{ACCORD SUR LA PROTECTION DES INVESTISSEMENTS}

En septembre 1981, la Suisse et le Sri Lanka ont signé un accord sur le développement mutuel et la protection des investissements. Cet acte fut passé durant la présence au Comptoir suisse, à Lausanne, du ministre du commerce et de la navigation du Sri Lanka. L'accord est entré en vigueur le 12 février 1981. Lors de la visite en Suisse du président de l'Etat ghanéen, en octobre 1981 , des conversations ont été engagées au sujet d'un accord bilatéral de protection des investissements, mais elles n'ont pas abouti pour le moment (37).

Les accords bilatéraux sur la protection des investissements doivent dans toute la mesure du possible créer des conditions favorables aux investissements de capitaux; ils représentent l'un des instruments de la politique de développement de la Suisse. Leur fonction est en quelque sorte d'améliorer prudemment la sécurité de l'investissement. Leur efficacité est cependant freinée par les difficultés d'expression juridique, par le fait qu'ils portent surtout sur les investissements nouveaux et par le faible mouvement des investissements vers les pays intéressés. Ils s'appuient sur l'autorisation conférée à terme à la Confédération de conclure des accords de protection et de développement des investissements de capitaux, autorisation datée du 14 décembre 1973 (prolongation). Pour l'essentiel, ces accords portent sur les points suivants :

- traitement égal ou clause de la nation la plus favorisée : assurance que les investisseurs suisses seront traités sur un pied d'égalité avec les investisseurs nationaux et jouiront du statut de la nation la plus favorisée;

- libre transfert des revenus;

- indemnisation obligatoire en cas d'expropriation;

- règlement des conflits par un tribunal arbitral (38).

A la fin du premier semestre 1982 la Suisse avait conclu dix-sept traités bilatéraux de protection des investissements, tous avec des pays en développement. Le tableau suivant en donne la liste. 


\section{ACCORDS DE PROTECTION DES INVESTISSEMENTS SUISSES}

(au 30 juin 1982)

Date de la

Date de

signature

l'entrée en

vigueur

Accords de protection et de

développement des investissements

Tunisie

3.12.1961

19. 1.1964

Tanzanie

3. 5.1965

16. 9.1965

Costa Rica

1. 9.1965

18. 8.1966

Honduras

20. 7.1966

Equateur

2. 5.1968

11. 9.1969

Corée du Sud

7. 4.1971

7. 4.1971

Ouganda

23. 8.1971

8. 5.1972

Zaïre

16. 3.1972

10. 5.1973

République arabe d'Egypte

25. 7.1973

4. 6.1974

Indonésie

6. 2.1974

9. 4.1976

Soudan

17. 2.1974

14.12.1974

Jordanie

11.11 .1974

3. 3.1977

Syrie

23. 6.1977

10. 8.1978

Malaisie

1. 3.1978

9. 6.1978

Singapour

6. 3.1978

3. 5.1978

Mali

8. 3.1978

8.12.1978

Sri Lanka

23. 9.1981

12. 2.1982

Source : Office fédéral du commerce extérieur.

\section{PREFERENCES DOUANIERES}

En 1981, 13\% des produits importés des pays en développement ont été introduits en Suisse au bénéfice des préférences douanières. Au cours des deux dernières années, la proportion de ces importations a baissé par rapport au total des importations des pays en développement. Des articles beaucoup plus nombreux auraient pu bénéficier du traitement de faveur puisque les préférences douanières n'ont été utilisées qu'à 37\% de leur capacité en 1981 (39). On peut expliquer cette situation par la faible taxation des matières premières en général, par la baisse des tarifs douaniers décidés par le GATT 
Iors du Tokyo Round et par les libertés prises avec les règlements sur la provenance.

En 1981, les importations en provenance des pays en développement se sont élevées à 5,87 milliards de francs, soit $9,8 \%$ du total des importations suisses, alors que les exportations vers ces mêmes pays s'élevaient à 11,74 milliards de francs, soit $22,2 \%$ du total des exportations. I'excédent de la balance commerciale avec les pays en développement est donc de 5,97 milliards de francs.

Le délai référendaire s'étant écoulé sans être utilisé, l'arrêté fédéral sur l'octroi de préférences douanières aux pays en développement est entré en vigueur le 1er mars 1982. Le Conseil fédéral peut désormais accorder des préférences douanières durant dix nouvelles années. II peut,"aussi longtemps que les circonstances l'exigent, modifier ou suspendre les préférences tarifaires ou prendre toute autre mesure qu'il juge nécessaire". Et cela "si I'application des mesures tarifaires a, sur le trafic des marchandises, des effets tels que les intérêts économiques suisses essentiels s'en trouvent ou risquent de s'en trouver affectés, ou que des courants d'échanges sont fortement perturbés". Le Conseil fédéral examine périodiquement I'octroi des préférences douanières dans I'optique du développement, de l'équilibre financier et de la politique commerciale (40).

Le 26 mai 1982, le Conseil fédéral a décidé d'ouvrir l'éventail des préférences douanières pour les marchandises venant des pays en développement. Selon les nouvelles dispositions valables dès le 1er juillet 1982, les trente et un pays les moins avancés jouiront de l'exonération douanière de leurs exportations de biens industriels pour lesquels les autres pays en développement n'ont que des préférences limitées. Cette disposition concerne notamment les textiles, I'habillement, les chaussures et toute une série de produits agricoles (arachides, poudre de cacao, ananas). Le Conseil fédéral a en outre adapté les préférences douanières dans le cas d'autres produits en provenance des pays en développement.

Au 1er janvier et au 1er juillet 1982, le Conseil fédéral a modifié la réglementation sur les certificats d'origine. II a ainsi apporté, conformément aux vœux exprimés par les pays en développement, une contribution à I'harmonisation internationale de la réglementation sur les certificats d'origine (41). 
(E) әр \% иа (9)

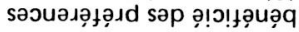

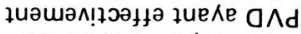

sәp әэиеนวлолd иә suO!]

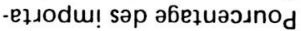

(G) auuo|Oo e| ę Lodded led (9) әuиo|о ए। әp

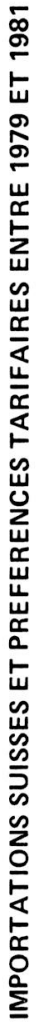
\% ua sopıoso sabezuene

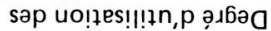

soueגf әр suo!!I! u uә sajuasaładd

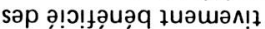

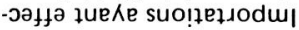

souexf ap suo!!I! u ua

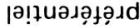

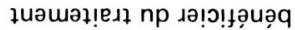
luesnod suo!jeztodm|

\section{융}

লે

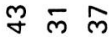
i i

(C) əuuojos el e łodded

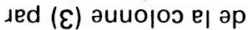

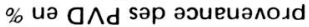
uә suo!lezlodu! sәp дее

$\hat{\sigma} \cong$

souedt әp suo!!I! u uә

(e (a^d) luawaddo

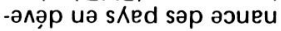
-ənodd uə suo!lezsodw|

$\widehat{\underline{m}}$

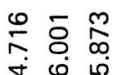

soueגf әp suo!!!!u ua ass!ns

е) ap suolfeflodu! sap ןejol aunjo^

일

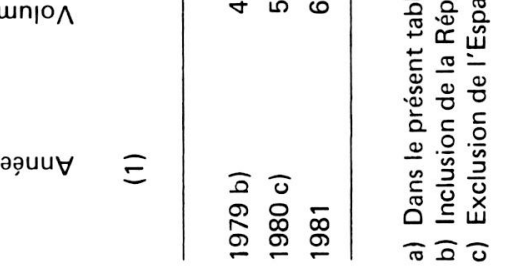




\section{CONSOLIDATION DES DETTES ET REECHELONNEMENT DES PAIEMENTS}

II faut signaler un accord avec la Sierra Leone concernant un rééchelonnement de dettes. Conclu le 2 juin, il est entré en vigueur le 30 juin 1981. II concerne des crédits d'exportation garantis par la Suisse et resté impayés, d'un montant de 3,4 millions de francs. Ces dettes seront remboursées du 30 juin 1981 au 30 juin 1991 en quatre annuités dans un premier temps, puis en douze tranches semestrielles. L'intérêt est de 6,5\% et doit être acquitté en francs suisses. La Sierre Leone s'engage en outre à ne pas soumettre la Suisse à un traitement moins favorable que celui qui est consenti à tout autre pays créancier (42).

Avec le Togo également, un accord de rééchelonnement de dettes a été conclu le 15 juin 1981. II porte sur un montant de 57 millions de francs. Le remboursement se fera sur une durée de neuf ans. En 1979 déjà, un semblable accord avait été conclu avec le Togo (43).

Le 10 septembre 1981, un accord de rééchelonnement de dettes a été conclu avec Madagascar. II est entré en vigueur le 18 mars 1982. II porte sur des crédits à l'exportation garantis par la Suisse et restés impayés, pour un montant de 2,5 millions de francs. Ces dettes seront remboursées en grande partie par des versements semestriels échelonnés jusqu'à fin septembre 1990. Payables en francs suisses, les intérêts sont calculés à un taux annuel de $7,75 \%$. Madagascar n'accordera pas à la Suisse un traitement moins favorable qu'à tout autre pays créancier (44). Le 13 juillet 1982, les pays occidentaux membres du Club de Paris, créanciers de Madagascar, ont conclu un accord d'aménagement de la dette.

Le 20 janvier 1982, un accord bilatéral a été conclu sur le rééchelonnement de dettes du Sénégal envers la Suisse. Il est entré en vigueur le 10 juillet 1982. II porte sur des dettes d'un montant de 5,7 millions de francs résultant de l'octroi de crédits à l'exportation. Ces dettes devront être remboursées jusqu'à fin 1990, avec un intérêt de 7,5\% par an. D'autres dettes antérieures devront être remboursées jusqu'au 30 septembre 1982 (45).

Un autre accord de consolidation des dettes portant sur une somme de 19 millions de francs a été conclu le 22 août 1981 avec la République centrafricaine (46).

Treize Etats créanciers occidentaux réunis à l'échelon international en mars 1982 à Paris ont également convenu d'un plan d'aménagement des dettes du Soudan. Ce texte prévoit le remboursement de tous les crédits officiels ou garantis par l'Etat, ayant une validité de plus d'un an et arrivant à échéance entre le 1er juillet 1981 et le 31 décembre 1982. Le remboursement est à effectuer au cours des dix années qui viennent. Dans un communiqué commun, les Etats créanciers se déclarent satisfaits des mesures d'assainissement économi- 
que prises par le Soudan dans le cadre d'un programme du FMI (48).

\section{ENTRETIENS ECONOMIQUES}

Entre juillet 1981 et juin 1982, des représentants du gouvernement suisse et de l'Office fédéral des affaires économiques extérieures (OFAEE) ont eu à diverses reprises des entretiens sur l'économie avec des représentants de pays en développement. Nous évoquons brièvement les rencontres qui ont eu le plus de relief. Le 15 septembre 1981, une délégation algérienne a été reçue par le conseiller fédéral Honegger et de hauts fonctionnaires du Département de l'économie publique (DEP) (49). Le 25 septembre 1981, après une réunion de la commission de coopération économique Suisse - Corée du Sud, eurent lieu des conversations de portée économique et politique entre le ministre sud-coréen de l'industrie et du commerce, des membres du Conseil fédéral et de hauts fonctionnaires du DEP, les entretiens portant notamment sur la participation suisse à la construction d'usines de force motrice et à des “joint-ventures" en Corée du Sud (50).

Le 7 octobre 1981, il y eut entre des représentants de I'OFAEE et de l'économie jordanienne un échange de vues consacré entre autres à l'intensification de la coopération entre entreprises industrielles des deux pays (51). Du 6 au 8 octobre s'est tenue à Pékin la 5ème session de la commission économique mixte Suisse-Chine créée en vertu du traité de commerce en cours. L'orientation nouvelle de la politique économique chinoise va peut-être provoquer dans un proche avenir une stagnation des relations commerciales, mais la Chine reste intéressée par toutes les formes de la coopération industrielle (52).

Une délégation suisse dirigée par le secrétaire d'Etat Jolles et le président de la Banque nationale Leutwiler a fait du 9 au 24 novembre une visite officielle en Argentine, au Brésil et au Pérou. Elle montrait ainsi à quel point la Suisse souhaite fortifier ses échanges économiques avec ces pays, surtout en matière d'investissements et de transferts de technologie. Un membre de la délégation s'est en outre rendu à Santiago du Chili. La délégation inaugura en Argentine I'exposition "Tecno Suiza" et visita le pavillon suisse aux foires industrielles de Lima et de Santiago.

Dans une déclaration commune, la Suisse et le Pérou annoncent un regain de la coopération dans le domaine de l'aide au développement grâce à l'écoulement de produits péruviens sur le marché suisse et une montée des investissements, dans la branche de l'énergie et des transports notamment. L'intensification des échanges économiques va reposer d'une part sur l'octroi de préférences douanières et l'impulsion donnée aux importations, d'autres part sur l'octroi de la garantie aux risques de l'exportation. Un accord sur la double imposition est prévu et un crédit mixte est en chantier. 
Lors de la visite effectuée au Brésil, le projet minier de Carajas s'est trouvé au centre des conversations. Les investisseurs suisses pourraient participer à I'exploitation des gigantesques ressources minérales du bassin de l'Amazone (53).

Une délégation mixte formée de représentants de l'administration et de I'industrie a encore eu, en mars 1982, avec la Birmanie et les Philippines ainsi qu'avec des pays du golfe persique, des conversations touchant aux relations économiques bilatérales (54).

Par ailleurs, une délégation de l'Office suisse du tourisme (OST) a séjourné en novembre 1981 en diverses villes du Brésil et d'Argentine à des fins publicitaires. Son objectif était d'inciter les agences de voyage sud-américaines à tenir compte davantage de la Suisse dans leurs programmes. Les deux pays mentionnés fournissent la plus grande part des touristes sud-américains pénétrant en Suisse (on a compté environ 227.000 nuitées de Brésiliens et d'Argentins en Suisse au cours de 1981), alors que l'effectif des touristes en provenance des pays en développement représente une proportion d'à peu près $5 \%$ du total des visiteurs.

L'OST, une corporation de droit public de la Confédération, a pour mission d'organiser et de réaliser en Suisse et à l'étranger la propagande touristique au service de tout le pays. Cette propagande touristique nationale de portée mondiale doit écarter les risques d'une dépendance unilatérale par rapport à certains marchés. En termes de promotion régionale et de politique des devises, le tourisme compte beaucoup pour l'économie suisse. Par ses entrées en devises, il contribue notamment à équilibrer une balance commerciale traditionnellement passive. Dès 1983, la contribution que la Confédération accorde à I'OST passera de 15 à 21 millions de francs pour permettre à I'office de poursuivre sa mission. L'OST a deux agences dans des pays en développement, à Buenos-Aires et au Caire; il envisage d'en ouvrir une autre à Rio-de-Janeiro (55).

\section{EXPORTATIONS D'ARMES}

En 1981, les exportations suisses de matériel de guerre se sont élevées à 511,5 millions de francs (contre 340,6 millions de francs l'année précédente). L'augmentation par rapport à 1980 a donc été d'environ $50 \%$. Les ventes d'armements représentent presque $1 \%(0,97 \%)$ des exportations totales. Les pays en développement figurent pour $42 \%$ dans la liste des importateurs de matériel de guerre du fait d'une grosse commande isolée (fourniture au Nigéria de canons de DCA Oerlikon-Bührle). Parmi les clients principaux, on peut relever le Nigéria, Singapour, l'Argentine, le Pérou, les Philippines, I'Algérie et le Maroc actuellement engagé dans des opérations belliqueuses. 
La part de la Suisse à l'ensemble des livraisons internationales de matériel de guerre aux pays en développement est cependant faible. Le tableau suivant indique le volume des ventes suisses, classées par pays de destination (56).

Telle qu'elle est définie par la loi, l'exportation de matériel de guerre est soumise à l'autorisation du Conseil fédéral. 2.683 demandes dans ce sens ont été présentées en 1981, dont 2 seulement furent tranchées directement par le Conseil fédéral. Le nombre des demandes refusées n'est pas connu.

La fabrication à l'étranger sous licence ou les exportations effectuées par des entreprises suisses à partir de pays étrangers ne tombent pas sous le coup de la loi sur le matériel de guerre. C'est ainsi que la filiale italienne d'OerlikonBührle a reçu en 1981 une grosse commande égyptienne qui a bénéficié d'un crédit de 600 millions de francs fourni par des banques suisses à l'étranger. Au demeurant, un contrat de licence et une participation en capital de la même filiale sont en préparation pour la production d'armes au Brésil. A rappeler ici I'octroi d'une licence MOWAG au Chili en octobre 1980 pour la fabrication de véhicules blindés et le début de la production là-bas (57).

Les avions Pilatus-Porter des usines d'aviation Pilatus, à Stans, une filiale de Bührle, ne sont pas soumis à la loi sur le matériel de guerre. Ils ont de nouveau fait l'objet de controverses. 24 avions Pilatus ont été fournis en juillet 1981 à l'armée de l'air bolivienne, puis l'armée de l'air malaisienne a commandé 44 Pilatus-Porter PC-7 et le Mexique s'est intéressé aux Pilatus-Porter (PC-6) pour les doter éventuellement d'un armement. Une autre commande du Mexique est en cours de livraison; elle porte sur 55 appareils du type PC-7 (58).

Au Parlement, dans sa réponse à une question Ziégler (PS, GE), le Conseil fédéral a fait savoir le $\mathbf{2 4}$ mars que, selon les données fournies par l'ambassade suisse au Guatémala, I'armée de l'air guatémaltèque n'utilisait pas ces appareils au combat et qu'ils n'étaient pas armés. En mars 1982, I'un des mouvements de libération guetémaltèques (ORPA) a condamné l'emploi de ces appareils armés au cours de bombardements (59). Dans sa réponse à l'interpellation Morf (PS, ZH) sur l'emploi d'avions militaires suisses au Guatémala, le Conseil fédéral a relevé une fois de plus que les appareils Pilatus-Porter ne tombent pas sous le coup de la loi sur le matériel de guerre (60). 
EXPORTATIONS SUISSES DE MATERIEL DE GUERRE EN 1981

\begin{tabular}{|c|c|c|c|}
\hline PAYS & Total (en francs) & PAYS & Total (en francs) \\
\hline Nigéria & 149.969 .440 & Colombie & 258.450 \\
\hline Rép. féd. d'Allemagne & 143.732 .990 & Canada & 163.324 \\
\hline Autriche & 76.108 .960 & Afrique du Sud & 156.516 \\
\hline Suède & 44.554 .941 & Oman & 105.660 \\
\hline Grèce & 30.177 .759 & Yougoslavie & 67.886 \\
\hline Espagne & 17.724 .067 & Ghana & 66.719 \\
\hline Singapour & 9.631 .947 & Emirats arabes & 53.258 \\
\hline Pays-Bas & 8.311 .475 & Guinée & 44.039 \\
\hline France & 6.845 .712 & Jordanie & 40.179 \\
\hline Belgique/Luxembourg & 4.991 .701 & Brunei & 39.742 \\
\hline Italie & 2.894 .864 & Arabie saoudite & 38.264 \\
\hline Japon & 2.496 .949 & Turquie & 37.719 \\
\hline Philippines & 2.363 .316 & Inde & 27.120 \\
\hline Danemark & 2.282 .027 & Vénézuela & 13.717 \\
\hline Grande-Bretagne & 2.033 .648 & Côte-d'Ivoire & 12.575 \\
\hline Argentine & 1.697 .971 & Antilles néerlandaises & 11.835 \\
\hline Pérou & 1.235 .500 & Mexique & 8.652 \\
\hline Algérie & 919.240 & Bulgarie & 8.581 \\
\hline Finlande & 620.050 & Thaïlande & 7.333 \\
\hline Portugal & 532.227 & Australie & 6.496 \\
\hline Maroc & 476.406 & Roumanie & 5.093 \\
\hline Norvège & 392.042 & Brésil & 4.895 \\
\hline \multirow[t]{3}{*}{ USA } & 342.281 & Tansanie & 4.531 \\
\hline & & Divers & 103.914 \\
\hline & & TOTAL & 511.543 .090 \\
\hline
\end{tabular}

Source : Direction générale des douanes, Statistique du commerce extérieur suisse. 